\title{
AS MULHERES NAS ESCOLAS DE ENGENHARIA BRASILEIRAS: HISTÓRIA, EDUCAÇÃO E FUTURO
}

\author{
Carla Giovana Cabral ${ }^{1}$
}

\section{RESUMO}

Historicamente, as mulheres foram afastadas do círculo criativo e líder da produção científica e tecnológica. Isso limitou sua atuação fora da esfera privada da casa e foi, séculos após séculos, evidenciado pela sua ausểncia e condução em carreiras como física, quimica, biologia, matemática e engenharia.

O acesso das mulheres à leitura e à escrita, algo que começou em meados do século XVII, foi mudando seu lugar nas sociedades e, por conseqüểncia, sua participação em carreiras científicas e tecnológicas. Hoje, não há restrições aparentes para o seu acesso aos sistemas educacionais, mas ergue-se uma série de outras barreiras que restringem que participe mais da produção do conhecimento cientifico e tecnológico, hierárquica e territorialmente, num universo ainda predominantemente masculino de pesquisa e ensino. Embora tenha crescido o número de mulheres nos cursos de engenharia nos últimos anos, a média de professoras e pesquisadoras, em áreas como engenharia e ciência da computação, segundo o último censo do CNPq, é de $25 \%$.

Trabalhos realizados na Escola Politécnica da Universidade Federal do Rio de Janeiro, na Escola Politécnica da Universidade de São Paulo e no Centro Tecnológico da Universidade Federal de Santa Catarina, por exemplo, pesquisam a presença feminina na engenharia nos séculos $X X$ e XXI, recuperam a história das pioneiras e investigam questões sóciohistóricas e epistemológicas.

Como uma maior participação das mulheres na engenharia poderia contribuir para a construção de uma tecnologia mais voltada ao bem-estar das pessoas e uma educação tecnológica crítica que melhor prepare os engenheiros para os desafios contemporâneos?

Palavras-chave: Educação tecnológica; Mulheres na engenharia; Engenharia e sociedade; Ciência, tecnologia e gênero; História da ciência e da tecnologia.

\section{INTRODUÇÃO}

\author{
Stop. \\ $A$ vida parou \\ ou foi o automóvel? \\ Carlos Diummond de Andrade
}

A mitologia grega conta que Prometeu roubou o fogo de Hefesto, deus artesão, e o entregou aos homens, marcando assim o nascimento da tecnologia. Mas, se ao contrário do que cantam os poetas antigos, fosse a deusa Palas Atenas a autora da façanha? Teríamos outros sistemas e artefatos tecnológicos? Seriam os mesmos os valores empregados pelos cientistas e tecnólogos?

Historicamente, as mulheres foram afastadas do círculo criativo e líder da produção científica e tecnológica. Isso limitou sua atuação fora da esfera privada da casa e foi, séculos após séculos, evidenciado pela sua ausência e condução em carreiras como a física, a química, a biologia, a matemática, a engenharia e, mais recentemente, a computação. Estas áreas desenvolveram-

1 Universidade Federal de Santa Catarina - Programa de Pós-Graduação em Educação Científica e Tecnológica (doutorado) - Campus Universitário - Trindade - 88040-900 Florianópolis - Santa Catarina. E-mail: carla@ctc.ufsc.br 
se ao sabor de valores considerados masculinos, como a certeza, a eficiência, o controle e a ordem.

O acesso das mulheres à leitura e à escrita, algo que começou em meados do século XVII, foi mudando seu lugar nas sociedades (PERES SEDEÑO, 2001) e por conseqüência sua participação em carreiras cientificas e tecnológicas. Hoje, não há restrições aparentes para o acesso das mulheres aos sistemas educacionais, mas ergue-se uma série de outras barreiras que restringem sua participação na produção do conhecimento cientifico e tecnológico, hierárquica e territorialmente, num universo androcêntrico de pesquisa e trabalho.

Atualmente, no Brasil, a média de professoras e pesquisadoras, em áreas como a engenharia e a ciência da computação, não ultrapassa os $30 \%$. As mulheres estão mais presentes em áreas como letras, lingüística, artes e ciências humanas. O cenário é balizado por desigualdades e discriminação, diferenças de oportunidades e desprezo às peculiaridades do trabalho feminino.

Funestas guerras e a abissal diferença de dignidade e qualidade de vida entre os povos do mundo e mesmo dentro dos países - vide o Brasil - a humanidade se envolve obrigatoriamente em questionamentos sobre a condução das investigações científicas e da tecnologia (REES, 2003; BAZZO, LINSINGEN VALE PEREIRA, 2000). O desenvolvimento tecnológico tem sido conduzido para um desenvolvimento humano?

Neste ensaio, pretendemos apresentar alguns dados do censo realizado pelo Conselho Nacional de Desenvolvimento Cientifico e Tecnológico (CNPq) em 2002, especialmente os que se referem às áreas da Engenharia e da Ciência da Computação, nos seis estados com maior número de grupos de pesquisa, analisando-os no que respeita ao gênero.

Sem deixar de lembrar o clássico questionamento do porquê de tão poucas mulheres nessas áreas, alinhavamos reflexões sobre a construção do conhecimento científico e tecnológico sem a inclusão dos valores femininos, e quais as possíveis causas e conseqüências desse processo excludente para a humanidade.

Arrematando, nossas reflexões conduzem à necessidade de uma educação tecnológica calçada na perspectiva das relações entre ciência, tecnologia, sociedade (CTS) e gênero, linha da nossa pesquisa de doutorado.

O ensaio aqui traçado é parte da fase exploratória da pesquisa de tese, em que investigamos e reflitimos sobre os valores sócio-historicamente pelas mulheres e sua imbricação em histórias de vida e na construção do conhecimento científico e tecnológico. Trabalho em andamento está sendo realizado com professoras de engenharia e ciências da computação do Centro Tecnológico da Universidade Federal de Santa Catarina (CTC-UFSC).

\section{LINHAS TORTAS}

São pelo menos três os momentos-chave na história, segundo a pesquisadora espanhola Eulália Péres Sedeño (2001), em que as mulheres são de alguma forma recompensadas na luta pelo acesso à educação. O primeiro momento tem lugar no período entre o Renascimento e a Revolução Científica, meados do século XVIII, quando começam a aprender a ler e a escrever, mesmo sem a chancela da lei. Houve polêmica e questionava-se a capacidade intelectual 
das mulheres e se era adequado e mesmo conveniente que tivessem educação formal. Peres Sedeño (2001) assinala que de qualquer forma isto possibilitou que aparecessem publicações científicas e literatura voltadas para "damas", um universo das classes privilegiadas, no entanto.

Um século depois, a conquista foi pelo acesso ao ensino superior para todas as mulheres. Isto aconteceu em diferentes ocasiões nos paises ocidentais, até aproximadamente a primeira metade do século $X X$, quando também tem início o ingresso em academias de ciências, configurando um segundo momentochave de conquistas.

A partida para o terceiro momento se deu nos anos 60 do século $X X$, tempo de refletir quais foram os motivos pelos quais havia tão poucas mulheres estudando, trabalhando e liderando nas áreas científica e tecnológica, mesmo sem uma discriminação legal.

Este percurso histórico, entre outros, deu vazão a vários tipos de pesquisas sobre ciência, tecnologia e gênero, que se dedicam a revelar, discutir e abolir as diferenças, muitas vezes expressivas, entre homens e mulheres num mesmo espaço de atuação (CABRAL, 2003). Ensejam-se questöes envolvendo ciência, tecnologia e poder, o estatuto epistêmico do sujeito feminino, a recuperação, na história, das mulheres cientistas e tecnólogas e, dentre outras, mudanças em curriculos e motivação para que mais meninas e mulheres se interessem em aprender ciências ou cursar engenharias e computação, por exemplo.

Estudos recentes mostram cenários para as mulheres na ciência e na tecnologia em vários países do mundo. Londa Schienbinger (2001) critica com categoria a situação, questionando a influência do feminismo na ciência, e abrindo janelas de esperança quando aponta fatos positivos como um aumento no número de mulheres dirigentes de agências governamentais, a chefiar departamentos e em cadeiras acadêmicas de prestígio nos Estados Unidos da América do Norte. A pesquisadora não deixa de lembrar, entretanto, que "sociedades como a americana e a européia persistem no uso de divisões fundamentais entre vida doméstica e profissional, que datam do século XVIII" (SCHIENBINGER, 2001, p. 350).

Quer dizer que as mulheres continuam desempenhando seu papel tradicional de gênero: ser mães, esposas, cuidar das crianças, dos doentes e dos idosos (PÉRES SEDEÑO, 2001) - algo que deveria ser mais valorizado pela sociedade.

Cristina Santamarina, numa pesquisa realizada no final da década de 90 do século passado (2001), constatou que pouco mais de $50 \%$ dos alunos das universidades espanholas eram mulheres, sendo que $24 \%$ delas freqüentavam cursos de arquitetura ou engenharias técnicas; $27,7 \%$ estudavam em cursos superiores de engenharia. Embora não tenha publicado, no artigo examinado, o equivalente (percentual) de professoras e investigadoras na engenharia, ela consultou esse público para estudar seus valores e discursos. Tanto os professores quanto as professoras consideraram a engenharia uma carreira de perfil masculino, e a medicina, a psicologia e a enfermagem, por exemplo, de perfil feminino (SANTAMARINA, 2001).

Não quer dizer que uma carreira seja mais masculina ou feminina que outra, mas que os valores que as construiram têm imbricados a história e a cultura de homens e mulheres. Excluídas durante séculos da esfera pública, as mulheres viram apartados também o conjunto de seus valores, qualidades e características (EYNDE, 1994). 
O cenário não é muito diferente na América Latina (ATRIO et al, 2001), onde, todavia, convive-se com a ausência de estatísticas diferenciadas por sexo, uma prática mais corrente nos países desenvolvidos. Estudos que diagnosticam a presença das mulheres nas áreas cientificas e tecnológicas resultam em indicadores que permitem análises etárias e de gênero e de niveis hierárquicos, entre outras. Um estudo realizado em 1997 por pesquisadoras da Rede Argentina de Gênero, Ciência e Tecnologia (RAGCyT) revelou que, no Brasil, a posição das mulheres líderes, por idade e número total de investigadoras, resulta, em todas as faixas etárias, em menos da metade, exceto as mais jovens. Também se pôde constatar que a única disciplina em que as mulheres estavam em maioria era a de Ciências Humanas. Nas ciências agrárias, exatas e engenharias o predomínio era masculino. As autoras do trabalho concluíram que as barreiras não se manifestavam tanto no ingresso a estas carreiras, mas revelavam-se posteriormente, nos niveis intermediários e altos (ATRIO et al, 2001).

Um outro trabalho bastante relevante investigou a "presença feminina nas carreiras de ciência e tecnologia", dedicando atenção especial à Escola Politécnica da Universidade Federal do Rio de Janeiro (POLI-UFRJ) (TABAK, 2002), uma das precursoras do ensino de engenharia não-militar no Brasil (SILVA TELES, 1984). Significativo exemplo dá a autora na página 132: "não se registra uma única professora titular entre os 19 existentes" na Politécnica da UFRJ.

$\mathrm{Na}$ Escola Politécnica da Universidade de São Paulo (EPUSP), apenas $9 \%$ do corpo docente são mulheres. Dessas, duas são titulares, oito associadas e 38 doutoras (FACCIOTTI e SAMARA, 2004).

No Centro CTC-UFSC, dos 348 professores, há 51 mulheres, que representam menos de $15 \%$ do total - abaixo da média dos estados analisados, inclusive o de Santa Catarina - como se verá a seguir. Áreas como engenharia mecânica e engenharia elétrica, por exemplo, são as que têm menos professoras proporcionalmente ao total de docentes. Há também o caso do Departamento de Automação e Sistemas, que não tem sequer uma professora. Outra informação a ser considerada é a de que, em seus 43 anos de história, o CTC-UFSC não teve nenhuma diretora eleita. Helena Amélia Stemmer, do Departamento de Engenharia Civil, e Ana Maria de Mattos Juliano, do Departamento de Engenharia Química e Engenharia de Alimentos, ocuparam o cargo na condição de protempore. Na "Tabela 1", abaixo, mostra-se o quadro docente atual do CTC-UFSC.

Tabela 1 - QUADRO DOCENTE DO CTC-UFSC.

\begin{tabular}{l|c|c|c}
\hline Departamento & Mulheres & Homens & Total \\
\hline Arquitetura e Urbanismo & 14 & 31 & 45 \\
Automação e Sistemas & - & 18 & 18 \\
Engenharia Civil & 11 & 28 & 39 \\
Engenharia Elétrica & 4 & 42 & 46 \\
Engenharia Mecânica & 2 & 63 & 65 \\
Engenharia de Produção e Sistemas & 6 & 27 & 33 \\
Engenharia Quimica e Engenharia de Alimentos & 5 & 23 & 28 \\
Engenharia Sanitária e Ambiental & 2 & 15 & 17 \\
Informática e Estatística & 7 & 50 & 57 \\
\hline & 51 & 297 & 348 \\
\hline
\end{tabular}

Fonte: Sites dos departamentos e da Direção do Centro. 


\section{DISSONÂNCIAS}

Os dados apresentados pelo CNPq em seu censo 2002 mostram essa realidade ainda dissonante, territorial e hierarquicamente. Do total de 56.891 investigadores nas oito grandes áreas de pesquisa2, 30.859 são homem e 26.021 mulheres. Neste caso, a diferença não alarma, beira os $9 \%$. Avaliando-se a distribuição dos pesquisadores segundo a idade e o sexo (Figura 1, abaixo), nota-se que as mulheres são maioria apenas nas faixas etárias dos 24 aos 29 anos. Depois, gradativamente, começam a perder espaço. Dos 55 aos 65 anos, fase em que os pesquisadores podem trabalhar de forma mais madura e consciente seus projetos, acredita-se, há poucas mulheres, de 32 a $38 \%$ do total.

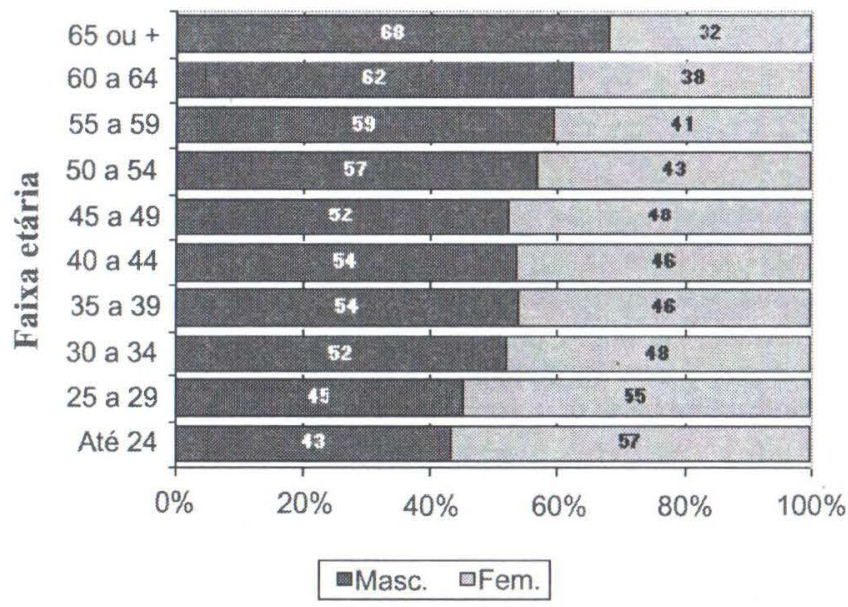

Figura 1 - Distribuição dos pesquisadores, segundo a faixa etária e o sexo. Fonte: CNPq. 2002.

A inquietação aumenta quando o olhar se volta para uma leitura hierárquica. A liderança das mulheres nas pesquisas somente existe na faixa etária até 24 anos (ver Tabela 2, a seguir), o que vai gradativamente diminuindo. Analisando a Figura 1 e a Tabela 2, verificamos que pode haver uma relação entre a quantidade de mulheres na área e a sua liderança. A leitura, porém, não pode ser linear, já que estudos comprovaram que um maior acesso de mulheres não alterou a hierarquia na área (ATRIO et al, 2001, SCHIENBINGER, 2001, TABAK, 2002). 
Tabela 2 - LIDERANÇANAS PESQUISAS POR FAIXAETÁRIAE SEXO.

\begin{tabular}{l|l|l|l|l|l}
\hline Faixa etária & Total & Homens & \% H & Mulheres & \% M \\
\hline Até $\mathbf{2 4}$ & 7 & 3 & 42,9 & 4 & 57,1 \\
$\mathbf{2 5}$ a $\mathbf{2 9}$ & 212 & 107 & 50,5 & 105 & $\mathbf{4 9 , 5}$ \\
$\mathbf{3 0}$ a $\mathbf{4 4}$ & 1.1 .51 & 686 & 59,6 & 465 & 40,4 \\
$\mathbf{3 5}$ a $\mathbf{3 9}$ & 3.222 & 1.979 & 61,4 & 1.243 & 38,6 \\
$\mathbf{4 0}$ a $\mathbf{4 4}$ & 3.959 & 2.268 & 58,1 & 1.634 & 41,9 \\
$\mathbf{4 5}$ a $\mathbf{4 9}$ & 4.367 & 2.464 & 56,4 & 1.903 & $\mathbf{4 3 , 6}$ \\
$\mathbf{5 0}$ a 54 & 3.959 & 2.371 & 59,9 & 1.588 & 40,1 \\
$\mathbf{5 5}$ a $\mathbf{5 9}$ & 2.396 & 1.434 & 59,8 & 962 & 40,2 \\
$\mathbf{6 0}$ a $\mathbf{6 4}$ & 1.119 & 695 & 62,1 & 424 & 37,9 \\
$\mathbf{6 5}$ ou mais & 723 & $\mathbf{4 8 1}$ & 66,5 & 242 & 33,5 \\
\hline Total & $\mathbf{2 1 . 0 6 2}$ & $\mathbf{1 2 . 4 9 2}$ & $\mathbf{5 9 , 3}$ & $\mathbf{8 . 5 7 0}$ & $\mathbf{4 0 , 7}$ \\
\hline
\end{tabular}

Fonte: CNPQ, 2002.

Pesquisadores da RAGCyT (ATRIO et al, 2001) vêm a idade como uma das variáveis que mais influenciam na baixa presença feminina nos cargos mais altos, considerando-se que atualmente vivemos um momento histórico de maior incorporação das mulheres nas áreas cientificas e tecnológicas.

As mesmas pesquisadoras crêem, no entanto, que a idade não é a razão fundamental, levantando como uma das hipóteses as "microdesiguadades". Seriam, como denominam as investigadoras, "comportamentos de exclusão geralmente insignificantes que passam inadvertidos, mas criam, ao se acumularem, um clima hostil que dissuade as mulheres a ingressar ou permanecer nas carreiras cientificas e tecnológicas" (ATRIO, et al, 2001, p. 37).

Tanto mais se dirige para regiōes e/ou estados, mais dissonâncias aparecem. Nos seis estados com maior número de grupos de pesquisa, as mulheres são maioria nas grandes áreas de ciências humanas $(60,56 \%)$, saúde (56,30\%) e letras, lingüistica e artes (69,51\%); nas ciências biológicas e sociais aplicadas, há uma distribuição mais eqüitativa; as engenharias e ciências da computação $(24,27 \%)$ e as ciências exatas e da terra $(29,62 \%)$ são as grandes áreas em que há os menores percentuais de mulheres (ver Gráfico 1), cujas grandes áreas preferenciais são ciências da saúde, humanas e biológicas (ver Gráfico 2). 
Gráfico 1 - DISTRIBUIÇÃO DE PESQUISADORES, POR SEXO, NAS OITO GRANDES ÁREAS DE CONHECIMENTO.

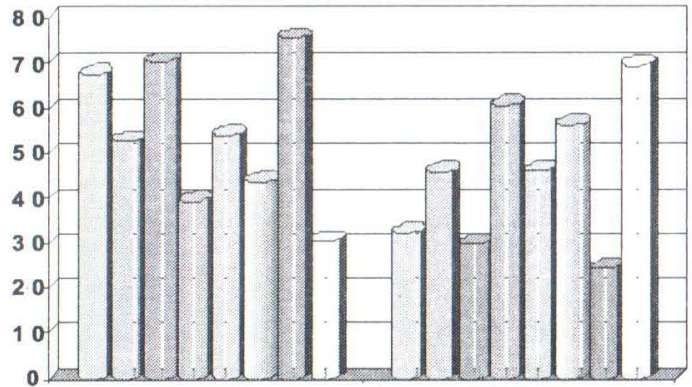

Agrárias

- Biológicas

Exatas

- Humanas

o Sociais

$\square$ Saúde

* Eng. E Comp.

$\square$ Letras

Homens

Mulheres

Fonte: CNPq, 2002

Gráfico 2 - DISTRIBUIÇÃO DE MULHERES NAS OITO GRANDES AREAS DE CONHECIMENTO.

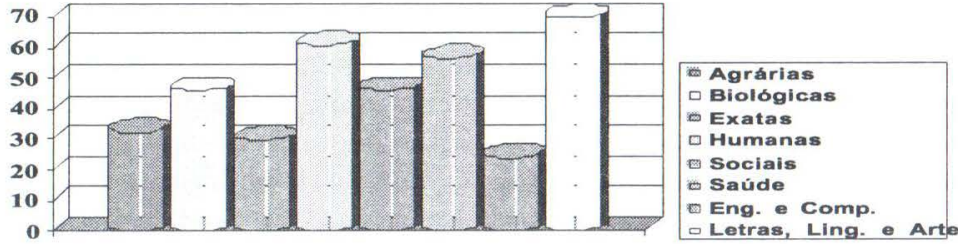

Fonte: CNPQf 2002. 
Fazendo uma leitura dos dados relacionados a engenharias e ciências da computação, quarta área com maior número de doutores no Pais, vamos encontrar uma diferença de cerca de dois terços, em prol dos homens. Os percentuais são semelhantes nos estados (ver Tabela 3).

Minas Gerais e São Paulo são as unidades da federação com o mais baixo percentual de mulheres - são pouco mais de $20 \%$.

\section{Tabela 3 - PESQUISADORES DA AREA DE ENGENHARIAS E CIÊNCIAS DA COMPUTAÇÃO.}

\begin{tabular}{l|l|l|l|l|l}
\hline ESTADO & Total & $\mathrm{H}$ & $\% \mathrm{H}$ & $\mathrm{m}$ & $\% \mathrm{M}$ \\
\hline SP & 3.124 & 2.433 & 77.88 & 691 & 22.11 \\
RJ & 1.476 & 1.061 & 71.88 & 415 & 28.11 \\
RS & 960 & 687 & 71.56 & 273 & 28.43 \\
MG & 868 & 685 & 78.91 & 183 & 21.08 \\
PR & 686 & 507 & 73.90 & 179 & 26.09 \\
SC & 768 & 596 & 77.60 & 172 & 22.39 \\
\hline TOTAL & $\mathbf{7 . 8 8 2}$ & $\mathbf{5 . 9 6 9}$ & & $\mathbf{1 . 9 1 3}$ & \\
\hline \multicolumn{2}{l|}{ MÉDIA } & Aprox. & & Aprox. \\
& & $\mathbf{7 5 \%}$ & & $\mathbf{2 5 \%}$ \\
\hline
\end{tabular}

Fonte: CNPq/ 2002

\section{REFLEXÕES INTRÉPIDAS}

Historicamente, as mulheres foram alheadas de um tipo de vida heróica, das façanhas célebres, dos jogos competitivos e da liderança de atividades de qualquer espécie (PACEY, 1990). Os trabalhos artesanais e o exercicio de atividades socialmente úteis como fiar e moer milho à mão ganharam a excelência feminina na Antigüidade, enquanto tarefas com fornos, fabricação de armas e construção de navios foram legados masculinos. De empreendimentos como esses derivam valores que Pacey (1990) chama de paralelos: (1) valentia e virtuosismo, de um lado; de outro, (2) necessidades básicas e o bem-estar humano. $\mathrm{A}$ arqueóloga Susan Walker assevera que moer grãos à mão não foi um trabalho feminino apenas no mundo de Homero, mas é algo que ocorre até hoje (WALKER, 1978, in PACEY, 1990). O pormenor é que, no momento em que se mecaniza a atividade agricola, como completa Pacey, são os homens que, então, se encarregam do trabalho.

A prática da tecnologia integra três tipos de valores: (1) de virtuosismo, (2) econômicos e (3) aqueles que refletem o trabalho realizado pelas mulheres (PACEY, 1990). O trabalho realizado pelas mulheres acabou thes atribuindo o lugar de usuária, mais do que produtora de tecnologia. A verdade é que dar um sentido econômico a um artefato ou sistema tecnológico não representa bem 
estar humano. Retomo a pergunta da introdução deste ensaio: o desenvolvimento tecnológico tem rumado para um desenvolvimento humano?

O desenvolvimento tecnológico que se assiste hoje é fruto de preceitos positivistas (BAZZO, LINSINGEN, VALE PEREIRA, 2003). Ou como disse o físico Marcelc Gleiser num domingo invernal de julho: "existem algumas áreas de pesquisa que podem acabar destruindo seus criadores e o resto da humanidade" (GLEISER, 2003, p. 18). Basta que aqui se cite a energia nuclear.

Um dos problemas que podemos aqui levantar em relação à construção do conhecimento tecnológico, com base no tripé de valores de Pacey (1990), é que a variável econômica cresceu de importância em demasia. Desta forma, o conceito de trabalho ganhou a característica de "produtivo" ou necessariamente produtivo para ser bom e válido. Relevando-se essa premissa, ignora-se o trabalho doméstico como trabalho real, pois "tem somente uso privado e carece de valor de troca" (ROSALDO, in PACEY, 1990, p. 170).

É importante prestar atenção à maneira como Pacey (1990) avalia o trabalho feminino. Ele diz que tem uma concepção diferente do significado da tecnologia, sem glorificar a construção da natureza, tampouco atende ao virtuosismo tecnológico.

Fugindo a uma visão mecanicista, as mulheres preocupariam-se mais com a conservação e a prevenção, do que com o controle, a ordem e a eficiência, por exemplo. Mas controle, ordem e eficiência foram justamente alguns dos mais fortes valores em que o conhecimento tecnológico foi gestado, adolescido e maturado. Tantas inovações tecnológicas, poucas inovações sociais e tão curto tempo entre um desenvolvimento e outro ...

Joan Rotschild (Rotschild, 1981, apud PACEY, 1990) crê que a perspectiva feminista (e nós inserimos aqui a feminina) pode ajudar na criação de um futuro tecnológico aprazivel, forjado com valores como a harmonia com a natureza e a não exploração. A pesquisadora vê essa construção como contrapeso.

Concordamos com essa perspectiva. Harmonia, prevenção, eliminação de riscos, conservação, solidariedade, compreensão, carinho não se opõem a controle, disciplina, eficiência, ordem, certeza, mas podem se complementar. Teriamos, então, um novo conceito de tecnologia?

Engenheiras(os) e cientistas da computação são tecnólogos por excelência. A engenharia, por exemplo, inclui, tanto a (1) inovação, o desenho e a construção, quanto a (2) operação, a manutenção e a utilização (PACEY, 1990). São aspectos ligados aos valores paralelos, de homens e mulheres. A face operacional de manutenção e utilização é, entretanto, subestimada.

Talvez haja a necessidade de engendrar os valores, pensar melhor a respeito dos conflitos entre o virtuosismo e da necessidade e os valores dos especialistas e usuários, como sugere Pacey (1990). É preciso que não se esqueça que o mercado inseriu demandas e que essas demandas podem estar sendo confundidas com valores. E os valores dos usuários?

Os valores nascem das aspirações pessoais, mas todos os indivíduos acabam vivenciando conflitos com os valores extrínsecos à sua própria experiência. O filósofo Hugh Lacey idealiza a existência de alguns fenômenos que ocorrem entre a aspiração e a realização dos indivíduos (LACEY, 1998); fenômenos que estão relacionados a brechas. Sem nos aprofundarmos nesta discussão filosófica, o que pretendemos em outro ensaio, entendemos brecha 
como um espaço de transformação, partindo da definição do próprio Lacey em relação ao fenômeno que se dá entre "a intenção e a ação efetiva, entre o desejo e os efeitos da ação" (LACEY, 1998, p. 48). Comumente, diz o filósofo, nossas ações não conduzem ao que pretendemos, e nossos desejos não são realizados por meio das ações que eles engendram. Cada um pode aqui pensar na sua experiência mesmo, na construção dos próprios valores. Transpondo essas breves reflexões a um episódio da história da ciência e da tecnologia como a do Projeto Manhatan, podemos razoavelmente imaginar que os cientistas e tecnólogos envolvidos nas pesquisas da energia nuclear e na construção das primeiras bombas atômicas não tivessem o desejo ou a intenção de aniquilar cidades e exterminar milhares de vidas. Ou teriam tido?

\section{CONSIDERAÇÕES FINAIS}

Talvez possamos pensar que a educação tecnológica atue nessa brecha como uma ação transformadora e capaz de dirimir ou acabar com as dissonâncias. Como Schienbinger (2001) questionou, se as mulheres fazem ciência diferente dos homens, podemos abrir um novo caminho e pensar que a tecnologia pode ser construída para o bem-estar de todos, por pessoas que abrigam e respeitam valores masculinos e femininos e compreendem o significado dessa construção.

A história pode ser (re)contada. Segundo a mitologia, a deusa Palas Atenas extrapolava a beleza e tinha qualidades técnicas e morais para o trabalho técnico e a perícia artesanal meticulosa. Mas como foi contada essa história?

Engendrar valores femininos na construção do conhecimento tecnológico, por isso, é como desdobrar um Prometeu às avessas.

\section{REFERÊNCIAS BIBLIOGRÁFICAS}

ATRIO, Jorge, FRANCHI, Ana, KOCHEN, Sitvia, MAFFIA, Diana. Situación de las mujeres en el sector científico-tecnológico en América Latina . In PERES SEDEÑO, E. (org) Las mujeres en el sistema de ciencia y tecnologia - estudios de casos. Madri: OEI, 2001. p. 19-39.

BAZZO, W. A., VALE PEREIRA, L. T., LINSINGEN, I. Educação tecnológica - enfoques para o ensino de engenharia. Florianópolis: EDUFSC, 2000.

BAZZO, W. A., LINSINGEN, I., VALE PEREIRA, L. T. Introdução aos estudos CTS (Ciência, Tecnologia e Sociedade). Madri: OEI, 2003.

CABRAL, Carla. "Educação científica e tecnológica + gênero = investigando o caráter situado do conhecimento". In: Encontro Nacional de Pesquisa em Ensino de Ciências, 6, 2003, São Paulo. Pôster.

CNPq. Censo 2002 do Conselho Nacional de Desenvolvimento Científico e Tecnológico. Disponivel em: <http://www.cnpq.br>.

EYNDE, Á. "Género y ciencia, ¿ términos condradictorios? Un análisis sobre la contribución de las mujeres al desarrollo cientifico". Revista Iberoamericana de Educación - gênero y educación, Madri, n. ${ }^{\circ} 6,1994$, p. 99. 
FACCIOTTI, M. C. R., SAMARA. E. M. As mulheres politécnicas: histórias e perfis. São Paulo: EPUSP, 2004.

GLEISER, M. Medo da ciência. Folha de São Paulo, 27 de julho de 2003.

LACEY, H. Para uma análise dos valores. In: LACEY, H. Valores na atividade cientifica. São Paulo: Discurso, 1998. p. 35-57.

PACEY, A. Las mujeres y los valores abiertos. In: PACEY. La cultura de la tecnologia. México: Fondade Cultura Económica, 1990.

PÉRES SEDEÑO, E. A modo de introducción: las mujeres en el sistema de cienciá y tecnología". In: PERES SEDEÑO, E. Las mujeres en el sistema de ciencia y tecnologia estudios de casos. Madri: OEI, 2001. p. 9-17.

REES, M. A explosão do humano. In: Folha de São Paulo, 25 de maio de 2003.

ROTSCHILD, J. A feminist perspective on technology and the future. In Women's Studies International Quaterly, 1981, apud PACEY, A. Las mujeres y los valores abiertos. In: La cultura de la tecnologia. México: Fondo de Cultura Económica, 1990.

SANTAMARINA, C. Las mujeres españolas ante el conocimiento centifico y tecnológico. In: PERES SEDEÑO, E. Las mujeres en el sistema de ciencia y tecnologia - estudios de casos. Madri: OEI, 2001. p. $41-57$.

SCHIENBINGER, L. O feminismo mudou a ciência? Bauru: Universidade do Sagrado Coração, 2001.

SILVA TELLES, P. Historia da engenharia no Brasil - do século XVI ao XIX. Rio de Janeiro: LTC, 1984.

TABAK, F. O laboratório de Pandora - estudos sobre a ciência no feminino. Rio de Janeiro: Garamond, 2002.

WALKER, S. Woman in antiquity, in SLATE, Londres: 1978, apud PACEY, A. Las mujeres y los valores abiertos. In: La cultura de la tecnologia. México: Fondo de Cultura Económica, 1990. 